\title{
Differences in Diffusion Tensor Imaging White Matter Integrity Related to Verbal Fluency Between Young and Old Adults
}

\author{
Benjamin Yeske ${ }^{* \star}$, Jiancheng Hou ${ }^{1,2 \dagger}$, Nagesh Adluru ${ }^{1,3}$, Veena A. Nair ${ }^{1}$ and \\ Vivek Prabhakaran ${ }^{1,4,5}$
}

${ }^{1}$ Department of Radiology, School of Medicine and Public Health, University of Wisconsin-Madison, Madison, WI, United States, ${ }^{2}$ Center for Cross-Strait Cultural Development, Fujian Normal University, Fuzhou, China, ${ }^{3}$ Waisman Center, University of Wisconsin-Madison, Madison, WI, United States, ${ }^{4}$ Department of Psychology, Department of Psychiatry, University of Wisconsin-Madison, Madison, WI, United States, ${ }^{5}$ Neuroscience Training Program, University

of Wisconsin-Madison, Madison, WI, United States

\section{OPEN ACCESS}

Edited by:

Toshiharu Nakai,

Osaka University, Japan

Reviewed by:

Yuto Uchida,

Nagoya City University, Japan

S. H. Annabel Chen,

Nanyang Technological University,

Singapore

*Correspondence:

Benjamin Yeske

byeske@wisc.edu

tThese authors have contributed equally to this work and share first

authorship

Received: 30 July 2021 Accepted: 14 October 2021 Published: 22 November 2021

Citation:

Yeske B, Hou J, Adluru N, Nair VA and Prabhakaran V (2021) Differences in Diffusion Tensor Imaging White Matter Integrity Related to Verbal Fluency Between Young and Old

Adults.

Front. Aging Neurosci. 13:750621. doi: 10.3389/fnagi.2021.750621
Throughout adulthood, the brain undergoes an array of structural and functional changes during the typical aging process. These changes involve decreased brain volume, reduced synaptic density, and alterations in white matter (WM). Although there have been some previous neuroimaging studies that have measured the ability of adult language production and its correlations to brain function, structural gray matter volume, and functional differences between young and old adults, the structural role of WM in adult language production in individuals across the life span remains to be thoroughly elucidated. This study selected 38 young adults and 35 old adults for diffusion tensor imaging (DTI) and performed the Controlled Oral Word Association Test to assess verbal fluency (VF). Tract-Based Spatial Statistics were employed to evaluate the voxel-based group differences of diffusion metrics for the values of fractional anisotropy (FA), mean diffusivity (MD), axial diffusivity (AD), radial diffusivity (RD), and local diffusion homogeneity $(\mathrm{LDH})$ in $12 \mathrm{WM}$ regions of interest associated with language production. To investigate group differences on each DTI metric, an analysis of covariance (ANCOVA) controlling for sex and education level was performed, and the statistical threshold was considered at $p<0.00083$ (0.05/60 labels) after Bonferroni correction for multiple comparisons. Significant differences in DTI metrics identified in the ANCOVA were used to perform correlation analyses with VF scores. Compared to the old adults, the young adults had significantly (1) increased FA values on the bilateral anterior corona radiata (ACR); (2) decreased $M D$ values on the right $A C R$, but increased $M D$ on the left uncinate fasciculus (UF); and (3) decreased RD on the bilateral ACR. There were no significant differences between the groups for AD or LDH. Moreover, the old adults had only a significant correlation between the VF score and the MD on the left UF. There were no significant correlations between VF score and DTI metrics in the young adults. This study adds to the growing body of research that WM areas involved in language production are sensitive to aging.

Keywords: diffusion tensor imaging (DTI), aging, tract-based spatial statistics (TBSS), white matter integrity, verbal fluency 


\section{INTRODUCTION}

Throughout adulthood, the brain undergoes an array of structural and functional changes during the typical aging process (Caserta et al., 2009). These changes involve decreased brain volume, reduced synaptic density, and alterations in white matter (WM; Masliah et al., 1993; Jernigan et al., 2001; Resnick et al., 2003). The structural deterioration of the brain is thought to be the reason for cognitive decline seen in the aging process; therefore, correlational studies comparing changes to brain structure and function are increasingly common. These neuroimaging studies have repeatedly shown age-related cortical network re-organization, specifically a reduction of hemispheric specialization toward more bilateral activation. This reduction of hemispheric specialization, known as the hemispheric asymmetry reduction in older adults (HAROLD) model (Cabeza, 2002), is well documented in studies using various imaging modalities, namely, electro-encephalography (Bellis et al., 2000), near-infrared spectroscopy (Herrmann et al., 2006), functional magnetic resonance imaging (fMRI; Cabeza, 2002; La et al., 2016), and diffusion tensor imaging (DTI; Ardekani et al., 2007). In addition to the reduction of hemispheric specialization, an anteroposterior gradient of the loss of WM integrity has also been observed, with the anterior regions of the brain being disproportionately affected in the aging process compared to the posterior regions (Pfefferbaum et al., 2005; Ardekani et al., 2007; Madden et al., 2009; Bennett et al., 2010; Sullivan et al., 2010).

Extensive research has been conducted to explore the aging declines seen in cognitive abilities, namely, working memory (Nyberg et al., 2012; Vaqué-Alcázar et al., 2020), executive function (Fjell et al., 2017; Webb et al., 2020), and language function (Wingfield and Grossman, 2006; Kantarci et al., 2011; Kemmotsu et al., 2012; Baciu et al., 2016). Previous neuroimaging studies concerning language function have measured the ability of adult language production and its correlations to brain function (Pihlajamäki et al., 2000), used fMRI to study language function (Wingfield and Grossman, 2006; Meinzer et al., 2009; Baciu et al., 2016), and studied structural gray matter volume involved in language function (Zhang et al., 2013); however, the structural role of WM in adult language production in individuals across the life span remains to be thoroughly elucidated.

One non-invasive MRI technique for in vivo mapping of the structures of WM is DTI, which provides detailed information on the underlying fiber tract architecture as reflected by diffusion patterns of water molecules (Sundaram et al., 2008; Pugliese et al., 2009). Fractional anisotropy (FA, a scalar measure of the directional constraint of water diffusion) and mean diffusivity (MD, the mean of three eigenvectors that each reflects separate directions of minimal and maximal diffusion) are the most frequently used metrics to investigate WM fiber tract integrity. More recently, studies on aging have included axial diffusivity $(\mathrm{AD}$, a scalar measure of diffusivity along the length of an axon; see Thomason and Thompson, 2011) and radial diffusivity $(\mathrm{RD}$, measure of water diffusion perpendicular to the axons and is associated with demyelination and neuro-inflammation with edema and macrophage infiltration; see Budde et al., 2011; Rayhan et al., 2013) in their analyses, as they are more specific to neural changes commonly involved in aging, namely, axonal damage or loss (AD; Song et al., 2003; Budde et al., 2007) and the degree of myelination (RD) (Song et al., 2002, 2003, 2005; Nair et al., 2005; Budde et al., 2007). Using these two metrics, some patterns have been identified to describe the differential aging associations in WM fiber tracts. For example, in some WM tracts, age-related decreases in FA are associated with increases only in RD, but not in AD (Bhagat and Beaulieu, 2004; Davis et al., 2009; Madden et al., 2009; Zhang et al., 2010). Other patterns observed are that decreases in FA are associated with significant increases in both $\mathrm{RD}$ and $\mathrm{AD}$ (Sullivan and Pfefferbaum, 2006; Zahr et al., 2009; Sullivan et al., 2010) and decreases in FA are associated with increases in RD and decreases in AD (Bennett et al., 2010). These patterns suggest that there may be differential aging processes occurring in different brain regions. Currently, these patterns remain unstudied in their relationship to age-related language production. Additionally, a novel inter-voxel metric called local diffusion homogeneity $(\mathrm{LDH})$, which quantifies the local coherence of water molecule diffusion in a model-free manner, was also examined in our analyses (Gong, 2013). Using the LDH metric to describe the WM fiber tracts is still in its infancy and several studies have reported it as being complementary to FA and MD in detecting changes in WM (Gong, 2013; Liu et al., 2017; Liang et al., 2019).

Currently, there are no studies using LDH to assess language production or brain aging more broadly; however, there are a handful of studies that have taken advantage of other DTI metrics to assess the structural role of WM in typically aging adults and language production. Stamatakis et al. (2011) identified that FA of the superior longitudinal fasciculus (SLF) and inferior longitudinal fasciculus (ILF) was positively correlated with accuracy in naming famous individuals. Madhavan et al. (2014) observed increased FA values on the SLF and increased age were positively associated with the performance in verbal fluency (VF) and word retrieval, respectively. They also identified a relationship between gender and FA values on the SLF tract and reported a linear decrease in FA in males and increase in FA in females until age 40, followed by a gradual decline. Houston et al. (2019) reported the performance on a wordretrieval task was associated with increased FA within the inferior fronto-occipital fasciculus (IFOF), in addition to the SLF, and observed increased FA within the corpus callosum that was associated with lower VF scores. Troutman and Diaz (2020) observed among all adult age groups that better performance on a naming with distractors task was associated with lower $\mathrm{RD}$ across dorsal, ventral, and fronto-striatal tracts as well as higher FA along dorsal tracts but was unable to find an association when covarying for age groups. Teubner-Rhodes et al. (2016) looked at age-related performance in acquisition and retrieval of lexical and semantic information and found agerelated declines in arcuate fasciculus (AF) microstructure were related to cognitive processing speed, but not to vocabulary retrieval. Interestingly, $\mathrm{FA}$ on the left $\mathrm{AF}$ was significantly 
related to individual variability in vocabulary independent of age, suggesting that the orientation and organization of the AF tract are stable with aging (Teubner-Rhodes et al., 2016). Taken together, these studies suggest that the dorsal stream pathway, specifically the SLF, may have a significant contribution to age-related differences in language production, but further research is needed.

Other tracts have also been identified to be key language comprehension and production pathways by studying patients suffering from diseases that afflict language function, namely, aphasia and strokes. In these patient populations, several WM tracts have been identified for being involved in language comprehension, namely, the IFOF (Ivanova et al., 2016; Hula et al., 2020), uncinate fasciculi (UF; Hula et al., 2020), middle longitudinal fasciculi (Hula et al., 2020), corona radiata (Grönholm et al., 2016; Sul et al., 2019), and external capsule (EC; Chen et al., 2015), and in language production, namely, the AF (Ivanova et al., 2016), middle longitudinal fasciculi (Hula et al., 2020), corona radiata (Grönholm et al., 2016; Sul et al., 2019), and EC (Chen et al., 2015). It is evident that injury to the aforementioned areas is known to affect language function; however, it is less understood how these regions are affected by the typical aging process and what effect this process has on language function.

This study employs Tract-Based Spatial Statistics (TBSS) to examine the regional brain differences related to language function (see the "Region of Interest Selection" in the "Materials and Methods" section for detailed regions) between young and old adults. TBSS is applied to perform automated analysis of WM integrity. TBSS uses a fine-tuned nonlinear registration method followed by a projection onto a mean FA skeleton. This skeleton represents the centers of all tracts common to the group and the resulting data fed into voxel-wise cross-subject statistics. Thus, TBSS combines the strength of both voxel-based and tractographic analyses to overcome the limitations of conventional methods, namely, standard registration algorithms and spatial smoothing (Dunst et al., 2014). Moreover, TBSS is assumed to improve the sensitivity, objectivity, and interpretability of multi-subject diffusion imaging studies (Smith et al., 2006; Dunst et al., 2014).

The VF task, which has traditionally been administered as a clinical neuropsychological paradigm to assess linguistic and executive function abilities, was used as behavioral testing in this study; it is one of the most widely used paradigms because of its simplicity and ease of administration. The cognitive components assessed by the VF task include executive functions, namely, initiation, inhibition, planning, updating, and shifting as well as verbal long-term memory (word knowledge) and lexicalsemantic linguistic processes (Shao et al., 2014).

Using group differences seen on WM metrics (i.e., FA, MD, $\mathrm{AD}, \mathrm{RD}$, and $\mathrm{LDH}$ ) in old vs. young adults, as well as group performance on the VF task, we hope to elucidate the impact aging has on the WM integrity and its relationship to language function. Portions of this manuscript have been previously presented (Hou et al., 2018).

\section{MATERIALS AND METHODS}

\section{Participants}

Notably, 38 young adults (21 men and 17 women, mean age $=23.58 \pm 3.35$ years $)$ and 35 old adults (19 men and 16 women, mean age $=60.91 \pm 5.25$ years) were recruited from the Madison, Wisconsin, campus community. They were free of any medical, neurological, or psychiatric disorders and had at least 14 years of education. A subset of the participants $(n=44)$ received the Mini Mental State Examination (MMSE; Folstein et al., 1975) and had scores $\geq 29$. For participants with educational level of high school graduate, a score on the MMSE of $\leq 25$ was considered cognitively impaired. The Edinburgh Handedness Inventory (Oldfield, 1971) was administered to all participants. A score greater than +40 was considered right-handed, between -40 and +40 was considered ambidextrous, and less than -40 was considered left-handed. Based on these criteria, there were 64 right, 7 left, and 2 ambidextrous in the study sample. Table 1 provides the basic demographic information of participants. All participants provided written informed consent. The experimental protocols were approved by the Institutional Review Board (IRB) of the School of Medicine and Public Health, University of WisconsinMadison.

\section{Behavioral Testing}

We administered the phonemic VF task [the Controlled Oral Word Association Test (COWAT); Benton and Hamsher, 1976] to test cognitive function. The COWAT has been extensively used in both clinical and non-clinical populations because of its face validity (Sauzéon et al., 2011), assessment

TABLE 1 | Demographic data and differences of VF testing.

\begin{tabular}{|c|c|c|c|c|}
\hline Characteristics & Young & Old & $t$ & $p$ \\
\hline Number & 38 & 35 & & \\
\hline Age (years) [range] & 23.58 (3.35) [18-32] & 60.91 (5.25) [55-77] & 28.64 & 0.000 \\
\hline Education (years) [range] & 16.79 (2.22) [12-21] & $17.43(2.87)[12-22]$ & 1.068 & 0.289 \\
\hline Sex (male/female) & $21 / 17$ & $19 / 16$ & 0.678 & 0.878 \\
\hline VF z-score & $-0.28(1.03)$ & $0.35(1.23)$ & 2.355 & 0.021 \\
\hline Handedness (right/left/amb) & $33 / 3 / 2$ & $31 / 4 / 0$ & 8.574 & 0.127 \\
\hline
\end{tabular}

Standard deviations are shown in parentheses. VF, verbal fluency; amb, ambidextrous. 
of both verbal cognitive ability and executive control (Fisk and Sharp, 2004), and high correlation with measures of attention, verbal memory, and word knowledge (Ruff et al., 1997). Participants were required to produce words beginning with the letters "F" "A," and " $\mathrm{F}$ " in three 1-min trials, respectively. Raw VF scores were based on the total correct responses over the three trials, which were then used to compute age and education corrected VF $\mathrm{z}$-scores based on a normative database (Tombaugh et al., 1999). This corrected
VF $\mathrm{z}$-score was used to quantify performance of VF for each participant.

\section{Magnetic Resonance Imaging Data Acquisition}

Diffusion-weighted images were acquired using a spin-echo based, single-shot, echo-planar diffusion sequence lasting $10 \mathrm{~min}$ on a GE750 $3 \mathrm{~T}$ MRI scanner. The specific parameters of

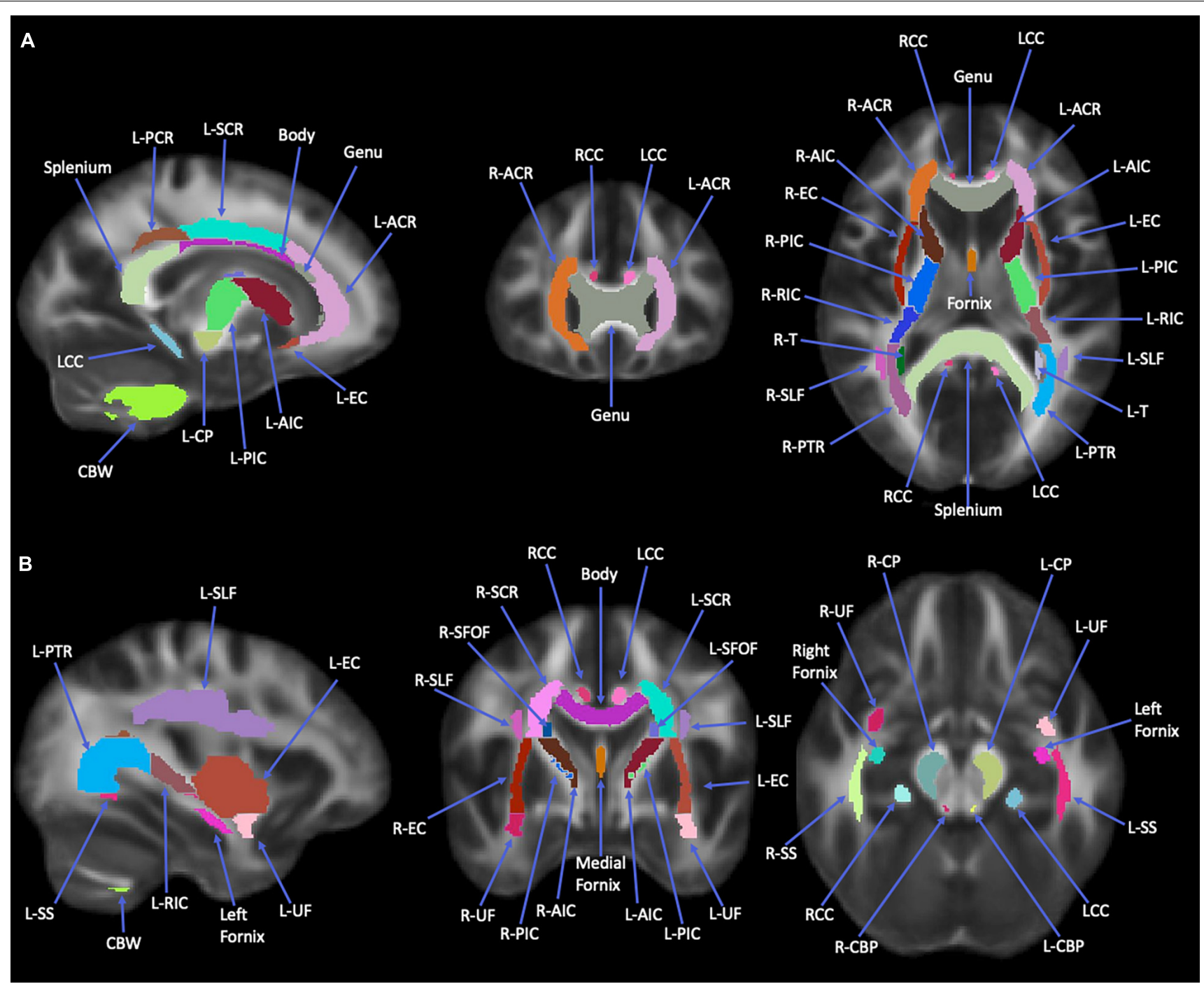

FIGURE 1 | Images of Johns Hopkins University (JHU)-ICBM-DTI-81 white matter (WM) atlas. This atlas was used to obtain the 12 regions of interest used in study analyses. Images are all WM tracts available in this atlas. (A) Orientations from left to right: sagittal, coronal, axial. (B) Orientations from left to right: sagittal, coronal, axial. Splenium, splenium of corpus callosum; Body, body of corpus callosum; Genu, genu of corpus callosum; L-ACR, left anterior corona radiata; R-ACR, right anterior corona radiata; L-SCR, left superior corona radiata; L-PCR, left posterior corona radiata; RCC, right cingulum cortex; LCC, left cingulum cortex; L-EC, left external capsule; L-AIC, left anterior limb of internal capsule; R-AIC, right anterior limb of internal capsule; L-PIC, left posterior limb of internal capsule; R-PIC, right posterior limb of internal capsule; L-RIC, left retrolenticular part of internal capsule; R-RIC, right retrolenticular part of internal capsule; L-CP, left cerebral peduncle; L-PTR, left posterior thalamic radiation; R-PTR, right posterior thalamic radiation; L-T, left tapetum; R-T, right tapetum; CBW, cerebellar white matter; L-UF, left uncinate fasciculus; R-UF, right uncinate fasciculus; R-EC, right external capsule; L-SLF, left superior longitudinal fasciculus; L-SS, left sagittal stratum; R-SS, right sagittal stratum; R-SCR, right superior corona radiata; L-SFOF, left superior fronto-occipital fasciculus; R-SFOF, right superior fronto-occipital fasciculus; R-CP, right cerebral peduncle; L-CBP, left cerebellar peduncle; R-CBP, right cerebellar peduncle. The regional masks were filtered with the white matter skeleton mask from TBSS (see Figure 2). 
MRI were as follows: repetition time $(\mathrm{TR})=9,000 \mathrm{~ms}$; echo time $(\mathrm{TE})=76.6 \mathrm{~ms}$; single average $(\mathrm{NEX}=1)$; field of view $=100 \mathrm{~mm} \times 100 \mathrm{~mm}$; matrix size $=256 \times 256$; inplane resolution $=1 \mathrm{~mm} \times 1 \mathrm{~mm} ; 75$ axial slices with no gap between slices and slice thickness $=2 \mathrm{~mm}$; excitation flip angle $\alpha=90^{\circ} ; 56$ gradient encoded directions $b$-value $=1,000 \mathrm{~s} / \mathrm{mm}^{2}$, 10 volumes with $b$-value $=0 \mathrm{~s} / \mathrm{mm}^{2}$. A high-resolution threedimensional T1-weighted BRAVO, IR-prepared Fast Spoiled Gradient Echo (FSPGR), MRI sequence with 156 axial slices was performed for each participant using the following parameters: $\mathrm{TR}=8.132 \mathrm{~ms} ; \mathrm{TE}=3.18 \mathrm{~ms}$, inversion time $(\mathrm{TI})=450 \mathrm{~ms}$; field of view $=256 \mathrm{~mm} \times 256 \mathrm{~mm}$; matrix size $=256 \times 256$; in-plane resolution $=1 \mathrm{~mm} \times 1 \mathrm{~mm}$; slice thickness $=1.0 \mathrm{~mm}$; excitation flip angle $\alpha=12^{\circ}$.

\section{Data Preprocessing}

All diffusion data were processed using the "Pipeline for Analyzing braiN Diffusion images" (PANDA): a toolbox implemented in MATLAB ${ }^{1}$ (Cui et al., 2013). This software employs several neuroimaging processing modules, namely, the FMRIB Software Library (FSL), the Pipeline System for Octave and Matlab (PSOM), the Diffusion Toolkit, and the MRIcron to automatically perform a series of steps (i.e., skull removal, correction of eddy current distortion, build diffusion tensor models) (Cui et al., 2013; Kashfi et al., 2017; Hou et al., 2020).

Diffusion metrics such as FA, MD, AD, RD (Smith et al., 2006; Smith and Nichols, 2009; Cui et al., 2013), and LDH (Gong, 2013) for each participant were extracted for 50 tracts identified from the Johns Hopkins University (JHU)-ICBM-DTI-81 WM atlas (Mori et al., 2008; Cui et al., 2013). Figures 1, 2 illustrate the representative images of WM tracts for this atlas. These region masks were filtered by applying the TBSS WM skeleton. Each global mean metric (i.e., FA, MD, $\mathrm{AD}, \mathrm{RD}$, and $\mathrm{LDH}$ ) for each participant was obtained by averaging across the 50 labels, with the diffusion metric for each label being divided by this global mean to account for any variability between participants. This standardized metric was used in our statistical analysis.

\section{Region of Interest Selection}

Based on prior studies on WM organization of the brain, six WM labels in each hemisphere implicated in language function (Smits et al., 2014; Friederici, 2015) were selected as regions of interests (ROIs), namely, ACR, superior corona radiata (SCR), posterior corona radiata (PCR), EC, UF, and SLF.

\section{Statistical Analysis}

A chi-square test was performed for handedness group differences, which did not identify a statistically significant difference between the two groups (see Table 1 for details). As a result, all handedness variations (right, left, and ambidextrous) were kept in the participant sample.

To investigate group differences on each DTI metric, an analysis of covariance (ANCOVA) controlling for sex and educational years was performed, and the statistical threshold for significance was considered at $p<0.00083$ (0.05/60 labels) after

\footnotetext{
${ }^{1}$ http://www.nitrc.org/projects/panda/
}

Bonferroni correction for multiple comparison. This analysis identified tracts in which the diffusivity metrics were significantly different between the groups. Pearson's correlation analysis, with sex as a covariate, was performed between the diffusivity metrics in the significant tracts and VF scores using IBM SPSS version 27 and considered significant at uncorrected $p<0.05$.

\section{RESULTS}

The VF testing demonstrated old adults have significantly higher VF $\mathrm{z}$-scores than young adults (see Table 1). Compared to the old adults, the young adults had significantly (1) increased FA values on the bilateral ACR; (2) increased MD value on the left UF; and (3) decreased RD on the bilateral ACR. There were no significant differences between the groups for $\mathrm{AD}$ or $\mathrm{LDH}$ (Table 2 and Figures 3, 4). Moreover, the old adults had only a significant positive correlation between the $\mathrm{VF} z$-score and the MD on the left UF $\left[r_{(35)}=0.383, p=0.025\right]$, with sex as a covariate (see Figure 5). There were no significant correlations between VF score and DTI metrics in young adults.

\section{DISCUSSION}

As reported previously in the literature, our study identified agerelated reductions in FA and increases in $\mathrm{RD}$ on the bilateral ACR as well as an increase in MD on the right ACR (Barrick et al., 2010; Bennett et al., 2010; Ly et al., 2014). Additionally, MD on the left UF showed significant group differences adding to the growing body of research that WM areas indicated in language function are sensitive to aging.

Hemispheric asymmetry with aging is well known (Cabeza, 2002). Specifically, the left lateralized language pattern seen in young adults changes with age to a more bi-hemispheric pattern in older adults, which could be a compensatory mechanism to

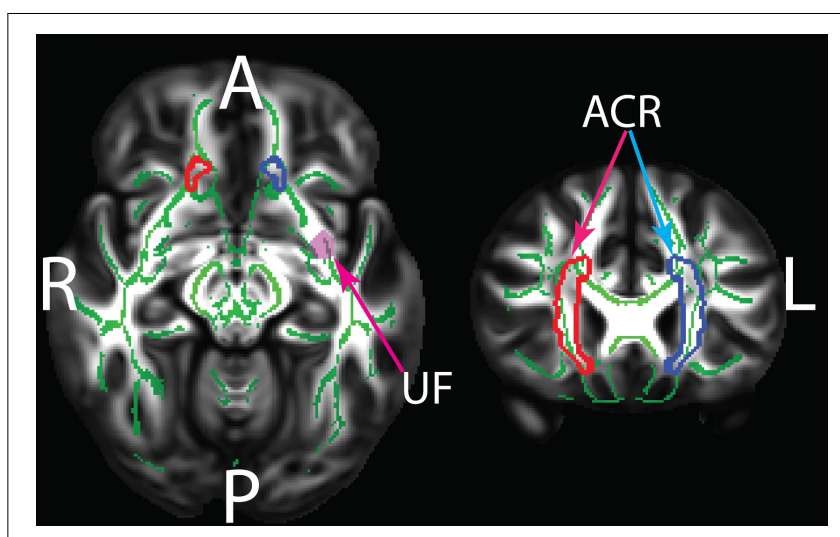

FIGURE 2 | The JHU white matter regions showing statistically significant group differences between old and young adults. ACR, anterior corona radiata; UF, uncinate fasciculus. The underlay shows the TBSS white matter skeleton which was masked by the JHU regions in the statistical analyses. The TBSS skeleton and the JHU regions are overlaid on the HCP 1065 FA template. Green: White matter skeleton; Red: right ACR; Blue: left ACR; pink: left UF. 
TABLE 2 | Significant difference between DTI metrics and tract by group comparisons.

\begin{tabular}{|c|c|c|c|c|c|c|}
\hline & \multirow[t]{2}{*}{ Tracts } & \multirow[t]{2}{*}{ Mean difference (young-old) } & \multirow[t]{2}{*}{ Standard error } & \multirow[t]{2}{*}{$p$} & \multicolumn{2}{|c|}{$95 \%$ confidence interval for difference } \\
\hline & & & & & Lower bound & Upper bound \\
\hline \multirow[t]{2}{*}{ FA } & Right ACR & 0.0454 & 0.0080 & 0.0000 & 0.0175 & 0.0732 \\
\hline & Left ACR & 0.0511 & 0.0083 & 0.0000 & 0.0221 & 0.0802 \\
\hline \multirow[t]{2}{*}{ MD } & Right ACR & -0.195 & 0.0053 & 0.0005 & -0.0380 & -0.0009 \\
\hline & Left UF & 0.0291 & 0.0078 & 0.0004 & 0.0018 & 0.0564 \\
\hline \multirow[t]{2}{*}{$\mathrm{RD}$} & Right ACR & -0.0415 & 0.0084 & 0.0000 & -0.0709 & -0.0121 \\
\hline & Left ACR & -0.0424 & 0.0094 & 0.0000 & -0.0752 & -0.0097 \\
\hline
\end{tabular}

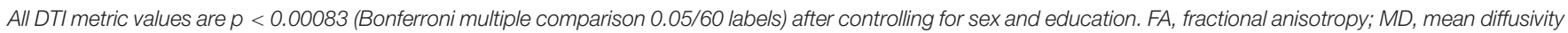
(unit: $\mu \mathrm{m}^{2} / \mathrm{ms}$ ); $R D$, radial diffusivity (unit: $\mu \mathrm{m}^{2} / \mathrm{ms}$ ); $A C R$, anterior corona radiata; UF, uncinate fasciculus.

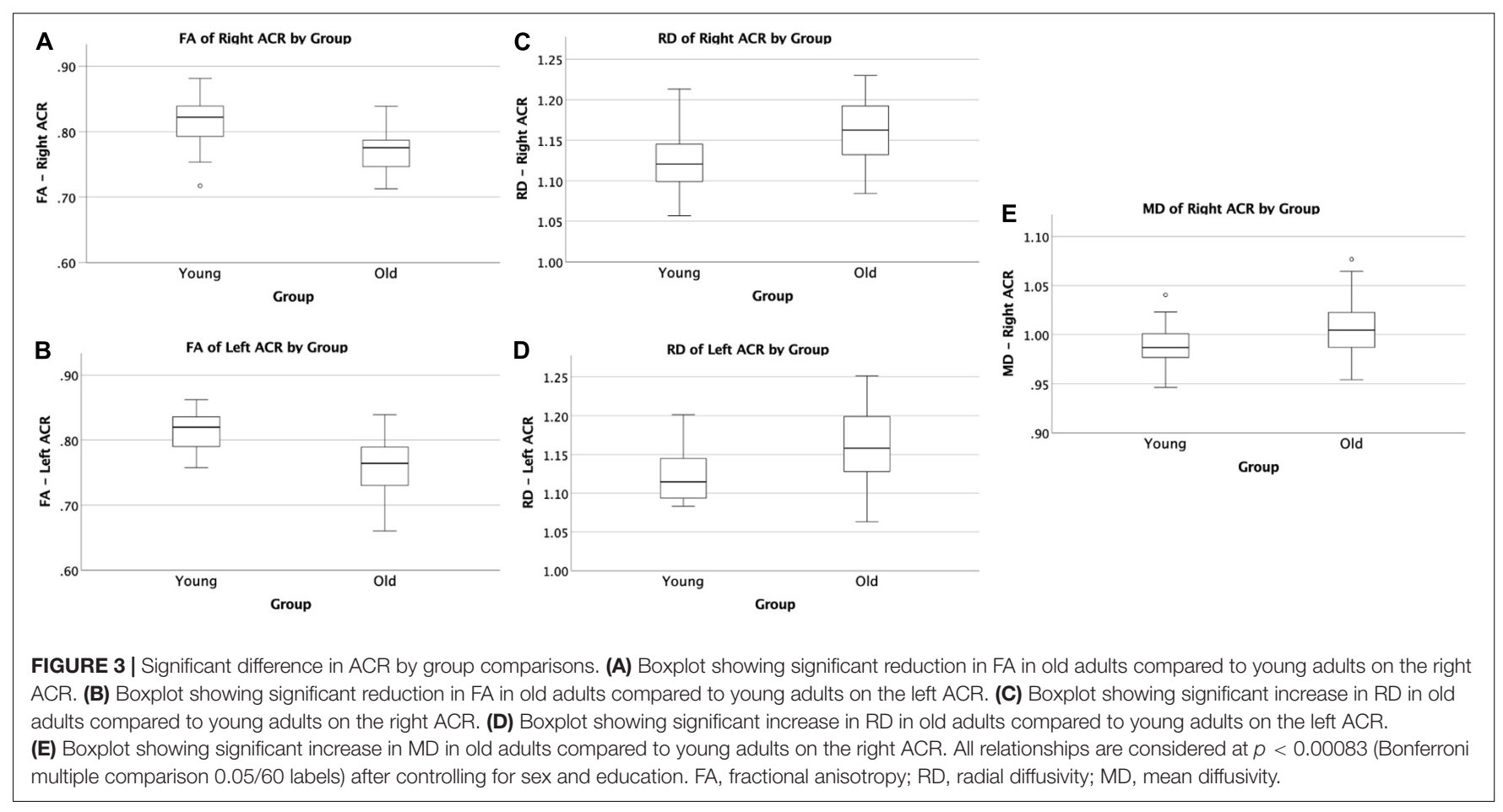

maintain behavioral performance. This study observed decreased MD on the left UF and increased MD on the right ACR in the old adults group, suggesting an improvement of the UF integrity and a decrease in the ACR. However, a decrease in left UF integrity would be predicted by the HAROLD model if the left hemispheric predominance of language function were to be reduced toward more bilateral activation (Cabeza, 2002). While this finding may seem to contradict the HAROLD model, it is difficult to assess the reduction of hemispheric specialization with only two nonbilateral group differences. Additionally, in this study, we only included one test of language/executive function. The asymmetry pattern is best investigated using a specific battery of language tests and will be explored in a future study.

This study did find evidence for another model of the aging brain, the anteroposterior model. Since the ACR resides more anteriorly than the other brain regions we studied, our findings for the ACR agree with prior evidence for the existence of an anteroposterior model for loss of WM integrity in the aging brain (Pfefferbaum et al., 2005; Ardekani et al., 2007; Madden et al.,
2009; Bennett et al., 2010). Since the UF is located inferiorly to the ACR, our findings of increased MD on the UF in old adults compared to young adults seem to contradict this assertion of anteroposterior aging. However, there is evidence to suggest that superiorly located fiber systems demonstrate age effects earlier than inferior systems (Sullivan et al., 2010) and this finding is additional evidence that there are brain regions with differential aging processes in aging adults. In addition, the significant increase in RD on the ACR signifies an age-related demyelination effect of that tract, based on previous animal and human studies investigating $\mathrm{RD}$ and its relationship to neural networks (Song et al., 2002, 2003, 2005; Nair et al., 2005; Budde et al., 2007), as well as evidence supporting typical aging is accompanied by myelin damage and loss (Peters, 2002). Our study did not find any association between ACR and VF, but Troutman and Diaz (2020) observed that higher $\mathrm{RD}$ across dorsal, ventral, and fronto-striatal tracts was associated with poorer performance on a naming with distractors task, suggesting that demyelination can result in poorer language performance. 


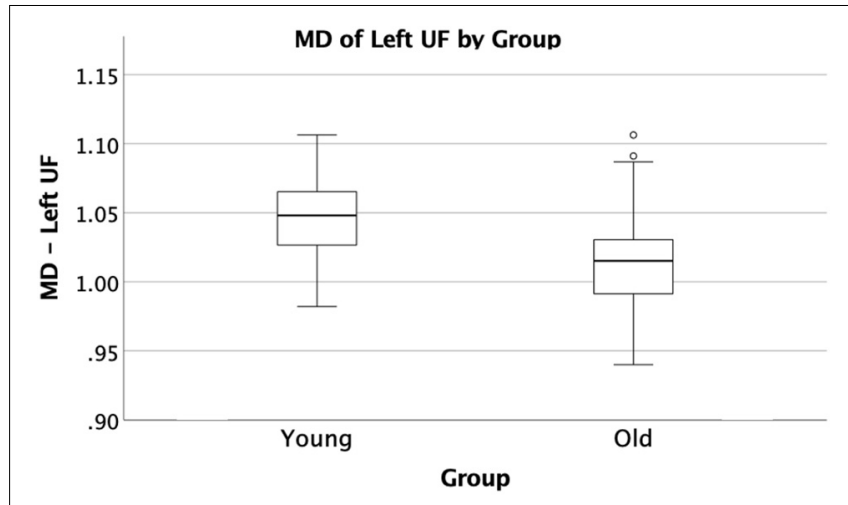

FIGURE 4 | Significant difference in UF by group comparison. Boxplot showing significant decrease in $\mathrm{MD}$ in old adults compared to young adults on the left UF. All relationships are considered at $p<0.00083$ (Bonferroni multiple comparison $0.05 / 60$ labels) after controlling for sex and education.

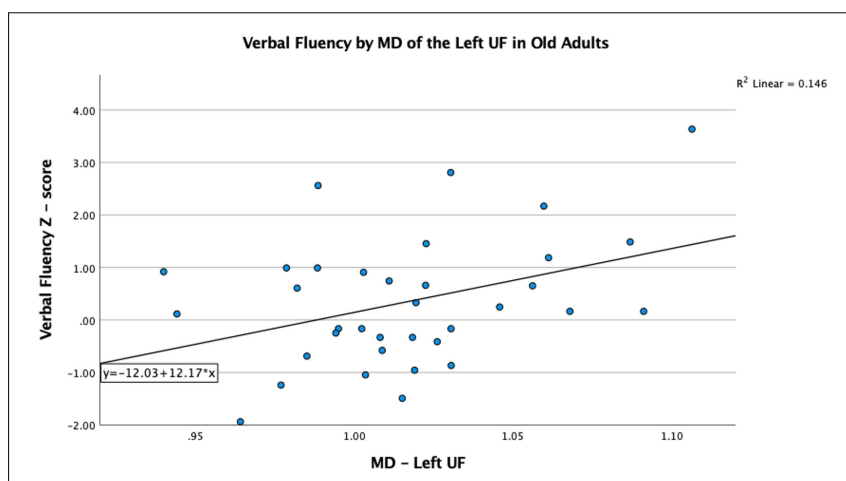

FIGURE 5 | Significant correlation between VF and MD on the left UF in older adults, after controlling for sex, $p<0.05$.

Compared to young adults, the significantly higher VF score we observed in the old adults group, in conjunction with the age-related demyelination and loss of WM integrity, could be evidence for the use of compensatory strategies to maintain performance. For instance, the HAROLD model purports that certain cognitive functions, namely, language production (La et al., 2016; Kim et al., 2018), lose their hemispheric specialization as one ages to counteract age-related neurocognitive deficits (Cabeza et al., 1997; Cabeza, 2002). Additionally, the compensation view of the HAROLD model has evidence to support recovery of language function after brain injuries (Cao et al., 1999) and resections (Jehna et al., 2017). Studies looking at temporal lobe resections in patients with epilepsy observed post-operative increases in FA in ipsilateral WM regions, such as the corona radiata and external and internal capsule, that were associated with a smaller fall in language proficiency after surgery (Yogarajah et al., 2010; Pustina et al., 2014). While age-related WM alterations may be different than the plastic changes occurring as a compensatory mechanism after regional brain insults, these studies still provide evidence that the brain has the capacity to rewire language pathways. Knowing this, perhaps age-related WM structural changes have a rewiring process to compensate for WM structures prone to degeneration allowing for preserved language function.
In our study, MD on the left UF of the old adult group was positively correlated with VF score, which is in contrast to what we would predict, since a higher MD typically indicates less WM integrity, and less WM integrity typically results in worse tract function. As a group, old adults have lower MD than young adults; however, within the old adult group, it is those with the higher MD values that score better on the VF task. Perhaps this indicates that the UF is becoming better organized to perform this task as we age, but only to a certain threshold, and perhaps the increased MD value in the older adults resulting in better performance is an indication of better compensation in those individuals by other WM tracts involved in VF performance. While we did not find other tract associations with the VF score that could attest to this postulation, Madhavan et al. (2014) did demonstrate a relationship with increased FA on the SLF and stronger language function in an aging population, suggesting some compensatory changes occurring in that tract. However, without other tract associations from this study, it is difficult to ascertain what the explanation for this may be, but future analyses involving a larger sample size may help elucidate this finding.

As previously mentioned, the demyelination and loss of WM integrity in the ACR, in addition to these UF findings, provides evidence that there may be differential aging processes in various WM tracts involved in language production that preserves this function with age. However, a few studies contradict this assertion by reporting losses of WM integrity in the UF with increasing age (Stamatakis et al., 2011; Kemmotsu et al., 2012; Gong, 2013). Taking this into account, it is difficult to interpret considering the sample sizes of the contradictory studies are all smaller than our current study. Further research will be needed to help resolve the discrepancy.

We did not identify any group differences for the AD and LDH DTI metrics, nor correlations of the VF score with the ACR, nor observe any significant group differences in the additional tracts we identified a priori. The lack of associations could have been a result of our small sample size and repeating this study with a larger population could elucidate more findings. While LDH is sensitive to diffusion properties among neighboring voxels and offers complementary information to $\mathrm{FA}, \mathrm{MD}$, and $\mathrm{RD}$, for this study, it is possible that the metric is not as sensitive to the brain aging process (Sullivan et al., 2001; Pfefferbaum et al., 2005; Sullivan and Pfefferbaum, 2006; Westlye et al., 2010; Lamar et al., 2014; Madhavan et al., 2014; Houston et al., 2019; Troutman and Diaz, 2020).

\section{DATA AVAILABILITY STATEMENT}

The original contributions presented in the study are included in the article/supplementary material, further inquiries can be directed to the corresponding author/s.

\section{ETHICS STATEMENT}

The studies involving human participants were reviewed and approved by the Institutional Review Board (IRB) of the School of Medicine and Public Health, University of Wisconsin-Madison. 
The patients/participants provided their written informed consent to participate in this study.

\section{AUTHOR CONTRIBUTIONS}

VP conceived and designed the experiments. VN helped with data acquisition. JH preprocessed the data and wrote the Materials and Methods section of the manuscript. BY and $\mathrm{JH}$ analyzed the data. BY wrote the Introduction, Results, and Discussion sections of the manuscript. VN, NA, and VP provided guidance for data analysis, and manuscript writing and editing. All authors contributed to the article and approved the submitted version.

\section{REFERENCES}

Ardekani, S., Kumar, A., Bartzokis, G., and Sinha, U. (2007). Exploratory voxelbased analysis of diffusion indices and hemispheric asymmetry in normal aging. Magn. Reson. Imaging 25, 154-167. doi: 10.1016/j.mri.2006.09.045

Baciu, M., Boudiaf, N., Cousin, E., Perrone-Bertolotti, M., Pichat, C., Fournet, N., et al. (2016). Functional MRI evidence for the decline of word retrieval and generation during normal aging. Age 38:3. doi: 10.1007/s11357-015-9857-y

Barrick, T. R., Charlton, R. A., Clark, C. A., and Markus, H. S. (2010). White matter structural decline in normal ageing: a prospective longitudinal study using tract-based spatial statistics. Neuroimage 51, 565-577. doi: 10.1016/j. neuroimage.2010.02.033

Bellis, T. J., Nicol, T., and Kraus, N. (2000). Aging affects hemispheric asymmetry in the neural representation of speech sounds. J. Neurosci. 20, 791-797.

Bennett, I. J., Madden, D. J., Vaidya, C. J., Howard, D. V., and Howard, J. H. (2010). Age-related differences in multiple measures of white matter integrity: a diffusion tensor imaging study of healthy aging. Hum. Brain. Mapp. 31, 378-390. doi: 10.1002/hbm.20872

Benton, A., and Hamsher, K. (1976). Multilingual Aphasia Examination. Iowa City: University of Iowa.

Bhagat, Y. A., and Beaulieu, C. (2004). Diffusion anisotropy in subcortical white matter and cortical gray matter: changes with aging and the role of CSFsuppression. J. Magn. Reson. Imaging 20, 216-227. doi: 10.1002/jmri.20102

Budde, M. D., Janes, L., Gold, E., Turtzo, L. C., and Frank, J. A. (2011). The contribution of gliosis to diffusion tensor anisotropy and tractography following traumatic brain injury: validation in the rat using Fourier analysis of stained tissue sections. Brain 134, 2248-2260. doi: 10.1093/brain/ awr161

Budde, M. D., Kim, J. H., Liang, H. F., Schmidt, R. E., Russell, J. H., Cross, A. H., et al. (2007). Toward accurate diagnosis of white matter pathology using diffusion tensor imaging. Magn. Reson. Med. 57, 688-695. doi: 10.1002/mrm. 21200

Cabeza, R. (2002). Hemispheric asymmetry reduction in older adults: the HAROLD model. Psychol. Aging 17, 85-100. doi: 10.1037//0882-7974.17.1.85

Cabeza, R., Grady, C. L., Nyberg, L., McIntosh, A. R., Tulving, E., Kapur, S., et al. (1997). Age-related differences in neural activity during memory encoding and retrieval: a positron emission tomography study. J. Neurosci. 17, 391-400.

Cao, Y., Vikingstad, E. M., George, K. P., Johnson, A. F., and Welch, K. M. (1999). Cortical language activation in stroke patients recovering from aphasia with functional MRI. Stroke 30, 2331-2340. doi: 10.1161/01.str.30.11. 2331

Caserta, M. T., Bannon, Y., Fernandez, F., Giunta, B., Schoenberg, M. R., and Tan, J. (2009). Normal brain aging clinical, immunological, neuropsychological, and neuroimaging features. Int. Rev. Neurobiol. 84, 1-19. doi: 10.1016/S00747742(09)00401-2

Chen, Y., Wang, A., Tang, J., Wei, D., Li, P., Chen, K., et al. (2015). Association of white matter integrity and cognitive functions in patients with subcortical silent lacunar infarcts. Stroke 46, 1123-1126. doi: 10.1161/STROKEAHA.115.008998

\section{FUNDING}

This study was supported by the NIH grants NINDS R01NS117568-01A1, RO1NS111022-01A1, R01NS105646, and NIMH RC1MH090912-01. NIH core grant Waisman Center from the National Institute of Child Health and Human Development (IDDRC U54 HD 090256) is also acknowledged. Funding for trainee, BY was provided by UW-Madison SMPH's Shapiro Summer Research Scholarship.

\section{ACKNOWLEDGMENTS}

Portions of this work were presented at the Organization of Human Brain Mapping Annual Meeting (OHBM) 2018.

Cui, Z., Zhong, S., Xu, P., He, Y., and Gong, G. (2013). PANDA: a pipeline toolbox for analyzing brain diffusion images. Front. Hum. Neurosci. 7:42. doi: 10.3389/ fnhum.2013.00042

Davis, S. W., Dennis, N. A., Buchler, N. G., White, L. E., Madden, D. J., and Cabeza, R. (2009). Assessing the effects of age on long white matter tracts using diffusion tensor tractography. Neuroimage 46, 530-541. doi: 10.1016/j.neuroimage.2009. 01.068

Dunst, B., Benedek, M., Koschutnig, K., Jauk, E., and Neubauer, A. C. (2014). Sex differences in the IQ-white matter microstructure relationship: a DTI study. Brain Cogn. 91, 71-78. doi: 10.1016/j.bandc.2014.08.006

Fisk, J. E., and Sharp, C. A. (2004). Age-related impairment in executive functioning: updating, inhibition, shifting, and access. J. Clin. Exp. Neuropsychol. 26, 874-890. doi: 10.1080/138033904905 10680

Fjell, A. M., Sneve, M. H., Grydeland, H., Storsve, A. B., and Walhovd, K. B. (2017). The Disconnected Brain and Executive Function Decline in Aging. Cereb. Cortex 27, 2303-2317. doi: 10.1093/cercor/bhw082

Folstein, M. F., Folstein, S. E., and McHugh, P. R. (1975). Mini-mental state". A practical method for grading the cognitive state of patients for the clinician. J. Psychiatr. Res. 12, 189-198. doi: 10.1016/0022-3956(75)90026-6

Friederici, A. D. (2015). White-matter pathways for speech and language processing. Handb. Clin. Neurol. 129, 177-186. doi: 10.1016/B978-0-44462630-1.00010-X

Gong, G. (2013). Local diffusion homogeneity (LDH): an inter-voxel diffusion MRI metric for assessing inter-subject white matter variability. PLoS One 8:e66366. doi: 10.1371/journal.pone.0066366

Grönholm, E. O., Roll, M. C., Horne, M. A., Sundgren, P. C., and Lindgren, A. G. (2016). Predominance of caudate nucleus lesions in acute ischaemic stroke patients with impairment in language and speech. Eur. J. Neurol. 23, 148-153. doi: $10.1111 /$ ene. 12822

Herrmann, M. J., Walter, A., Ehlis, A. C., and Fallgatter, A. J. (2006). Cerebral oxygenation changes in the prefrontal cortex: effects of age and gender. Neurobiol. Aging 27, 888-894. doi: 10.1016/j.neurobiolaging.2005.04.013

Hou, J., Dodd, K., Nair, V. A., Rajan, S., Beniwal-Patel, P., Saha, S., et al. (2020). Alterations in brain white matter microstructural properties in patients with Crohn's disease in remission. Sci. Rep. 10:2145. doi: 10.1038/s41598-02059098-w

Hou, J., Nair, V., Sinha, A., and Prabhakaran, V. (2018). "Differences in white matter integrity related to language production between young and old adults," in The 24th International Conference of Human Brain Mapping, (Singapore: Organization for Human Brain Mapping).

Houston, J., Allendorfer, J., Nenert, R., Goodman, A. M., and Szaflarski, J. P. (2019). White Matter Language Pathways and Language Performance in Healthy Adults Across Ages. Front. Neurosci. 13:1185. doi: 10.3389/fnins.2019.01185

Hula, W. D., Panesar, S., Gravier, M. L., Yeh, F. C., Dresang, H. C., Dickey, M. W., et al. (2020). Structural white matter connectometry of word production in aphasia: an observational study. Brain 143, 2532-2544. doi: 10.1093/brain/ awaa193 
Ivanova, M. V., Isaev, D. Y., Dragoy, O. V., Akinina, Y. S., Petrushevskiy, A. G., Fedina, O. N., et al. (2016). Diffusion-tensor imaging of major white matter tracts and their role in language processing in aphasia. Cortex 85, 165-181. doi: $10.1016 /$ j.cortex.2016.04.019

Jehna, M., Becker, J., Zaar, K., von Campe, G., Mahdy Ali, K., Reishofer, G., et al. (2017). Symmetry of the arcuate fasciculus and its impact on language performance of patients with brain tumors in the language-dominant hemisphere. J. Neurosurg. 127, 1407-1416. doi: 10.3171/2016.9.JNS161281

Jernigan, T. L., Archibald, S. L., Fennema-Notestine, C., Gamst, A. C., Stout, J. C., Bonner, J., et al. (2001). Effects of age on tissues and regions of the cerebrum and cerebellum. Neurobiol. Aging 22, 581-594. doi: 10.1016/s0197-4580(01) 00217-2

Kantarci, K., Senjem, M. L., Avula, R., Zhang, B., Samikoglu, A. R., Weigand, S. D., et al. (2011). Diffusion tensor imaging and cognitive function in older adults with no dementia. Neurology 77, 26-34. doi: 10.1212/WNL.0b013e31822313dc

Kashfi, K., Al-Khalil, K., Hou, J., Fang, D., Anderson, R., Rajmohan, R., et al. (2017). Hyper-brain connectivity in binge drinking college students: a diffusion tensor imaging study. Neurocase 23, 179-186. doi: 10.1080/13554794.2017.1347264

Kemmotsu, N., Girard, H. M., Kucukboyaci, N. E., McEvoy, L. K., Hagler, D. J., Dale, A. M., et al. (2012). Age-related changes in the neurophysiology of language in adults: relationship to regional cortical thinning and white matter microstructure. J. Neurosci. 32, 12204-12213. doi: 10.1523/JNEUROSCI.013612.2012

Kim, I. S., Millin, N. J., Hwang, J., Kim, I. S., Millin, N. J., and Hwang, J. (2018). Word retrieval by verbal fluency tasks for young and old people: an fNIR study. Clin. Arch. Commun. Disord. 3, 52-58.

La, C., Garcia-Ramos, C., Nair, V. A., Meier, T. B., Farrar-Edwards, D., Birn, R., et al. (2016). Age-Related Changes in BOLD Activation Pattern in Phonemic Fluency Paradigm: an Investigation of Activation, Functional Connectivity and Psychophysiological Interactions. Front. Aging Neurosci. 8:110. doi: 10.3389/ fnagi.2016.00110

Lamar, M., Zhou, X. J., Charlton, R. A., Dean, D., Little, D., and Deoni, S. C. (2014). In vivo quantification of white matter microstructure for use in aging: a focus on two emerging techniques. Am. J. Geriatr. Psychiatry 22, 111-121. doi: 10.1016/j.jagp.2013.08.001

Liang, Y., Zhang, H., Tan, X., Liu, J., Qin, C., Zeng, H., et al. (2019). Local Diffusion Homogeneity Provides Supplementary Information in T2DM-Related WM Microstructural Abnormality Detection. Front. Neurosci. 13:63. doi: 10.3389/ fnins.2019.00063

Liu, G., Tan, S., Dang, C., Peng, K., Xie, C., Xing, S., et al. (2017). Motor Recovery Prediction With Clinical Assessment and Local Diffusion Homogeneity After Acute Subcortical Infarction. Stroke 48, 2121-2128. doi: 10.1161/STROKEAHA. 117.017060

Ly, M., Canu, E., Xu, G., Oh, J., McLaren, D. G., Dowling, N. M., et al. (2014). Midlife measurements of white matter microstructure predict subsequent regional white matter atrophy in healthy adults. Hum. Brain Mapp. 35, 20442054. doi: $10.1002 / \mathrm{hbm} .22311$

Madden, D. J., Spaniol, J., Costello, M. C., Bucur, B., White, L. E., Cabeza, R., et al. (2009). Cerebral white matter integrity mediates adult age differences in cognitive performance. J. Cogn. Neurosci. 21, 289-302. doi: 10.1162/jocn.2009. 21047

Madhavan, K. M., McQueeny, T., Howe, S. R., Shear, P., and Szaflarski, J. (2014). Superior longitudinal fasciculus and language functioning in healthy aging. Brain Res. 1562, 11-22. doi: 10.1016/j.brainres.2014.03.012

Masliah, E., Mallory, M., Hansen, L., DeTeresa, R., and Terry, R. D. (1993). Quantitative synaptic alterations in the human neocortex during normal aging. Neurology 43, 192-197. doi: 10.1212/wnl.43.1_part_1.192

Meinzer, M., Flaisch, T., Wilser, L., Eulitz, C., Rockstroh, B., Conway, T., et al. (2009). Neural signatures of semantic and phonemic fluency in young and old adults. J. Cogn. Neurosci. 21, 2007-2018. doi: 10.1162/jocn.2009. 21219

Mori, S., Oishi, K., Jiang, H., Jiang, L., Li, X., Akhter, K., et al. (2008). Stereotaxic white matter atlas based on diffusion tensor imaging in an ICBM template. Neuroimage 40, 570-582. doi: 10.1016/j.neuroimage.2007.12.035

Nair, G., Tanahashi, Y., Low, H. P., Billings-Gagliardi, S., Schwartz, W. J., and Duong, T. Q. (2005). Myelination and long diffusion times alter diffusiontensor-imaging contrast in myelin-deficient shiverer mice. Neuroimage 28, 165-174. doi: 10.1016/j.neuroimage.2005.05.049
Nyberg, L., Lövdén, M., Riklund, K., Lindenberger, U., and Bäckman, L. (2012). Memory aging and brain maintenance. Trends Cogn. Sci. 16, 292-305. doi: 10.1016/j.tics.2012.04.005

Oldfield, R. C. (1971). The assessment and analysis of handedness: the Edinburgh inventory. Neuropsychologia 9, 97-113. doi: 10.1016/0028-3932(71)90067-4

Peters, A. (2002). The effects of normal aging on myelin and nerve fibers: a review. J. Neurocytol. 31, 581-593. doi: 10.1023/a:1025731309829

Pfefferbaum, A., Adalsteinsson, E., and Sullivan, E. V. (2005). Frontal circuitry degradation marks healthy adult aging: evidence from diffusion tensor imaging. Neuroimage 26, 891-899. doi: 10.1016/j.neuroimage.2005. 02.034

Pihlajamäki, M., Tanila, H., Hänninen, T., Könönen, M., Laakso, M., Partanen, K., et al. (2000). Verbal fluency activates the left medial temporal lobe: a functional magnetic resonance imaging study. Ann. Neurol. 47, 470-476.

Pugliese, L., Catani, M., Ameis, S., Dell'Acqua, F., Thiebaut de Schotten, M., Murphy, C., et al. (2009). The anatomy of extended limbic pathways in Asperger syndrome: a preliminary diffusion tensor imaging tractography study. Neuroimage 47, 427-434. doi: 10.1016/j.neuroimage.2009.05.014

Pustina, D., Doucet, G., Evans, J., Sharan, A., Sperling, M., Skidmore, C., et al. (2014). Distinct types of white matter changes are observed after anterior temporal lobectomy in epilepsy. PLoS One 9:e104211. doi: 10.1371/journal. pone. 0104211

Rayhan, R. U., Stevens, B. W., Timbol, C. R., Adewuyi, O., Walitt, B., VanMeter, J. W., et al. (2013). Increased brain white matter axial diffusivity associated with fatigue, pain and hyperalgesia in Gulf War illness. PLoS One 8:e58493. doi: 10.1371/journal.pone.0058493

Resnick, S. M., Pham, D. L., Kraut, M. A., Zonderman, A. B., and Davatzikos, C. (2003). Longitudinal magnetic resonance imaging studies of older adults: a shrinking brain. J. Neurosci. 23, 3295-3301.

Ruff, R. M., Light, R. H., Parker, S. B., and Levin, H. S. (1997). The psychological construct of word fluency. Brain Lang. 57, 394-405. doi: 10.1006/brln.1997. 1755

Sauzéon, H., Raboutet, C., Rodrigues, J., Langevin, S., Schelstraete, M. A., Feyereisen, P., et al. (2011). Verbal Knowledge as a Compensation Determinant of Adult Age Differences in Verbal Fluency Tasks over Time. J. Adult Dev. 18, $144-154$.

Shao, Z., Janse, E., Visser, K., and Meyer, A. S. (2014). What do verbal fluency tasks measure? Predictors of verbal fluency performance in older adults. Front. Psychol. 5:772. doi: 10.3389/fpsyg.2014.00772

Smith, S. M., Jenkinson, M., Johansen-Berg, H., Rueckert, D., Nichols, T. E., Mackay, C. E., et al. (2006). Tract-based spatial statistics: voxelwise analysis of multi-subject diffusion data. Neuroimage 31, 1487-1505. doi: 10.1016/j. neuroimage.2006.02.024

Smith, S. M., and Nichols, T. E. (2009). Threshold-free cluster enhancement: addressing problems of smoothing, threshold dependence and localisation in cluster inference. Neuroimage 44, 83-98. doi: 10.1016/j.neuroimage.2008.03. 061

Smits, M., Jiskoot, L. C., and Papma, J. M. (2014). White matter tracts of speech and language. Semin. Ultrasound CT MR 35, 504-516. doi: 10.1053/j.sult.2014. 06.008

Song, S. K., Sun, S. W., Ju, W. K., Lin, S. J., Cross, A. H., and Neufeld, A. H. (2003). Diffusion tensor imaging detects and differentiates axon and myelin degeneration in mouse optic nerve after retinal ischemia. Neuroimage 20, 1714-1722. doi: 10.1016/j.neuroimage.2003.07.005

Song, S. K., Sun, S. W., Ramsbottom, M. J., Chang, C., Russell, J., and Cross, A. H. (2002). Dysmyelination revealed through MRI as increased radial (but unchanged axial) diffusion of water. Neuroimage 17, 1429-1436. doi: 10.1006/ nimg. 2002.1267

Song, S. K., Yoshino, J., Le, T. Q., Lin, S. J., Sun, S. W., Cross, A. H., et al. (2005). Demyelination increases radial diffusivity in corpus callosum of mouse brain. Neuroimage 26, 132-140. doi: 10.1016/j.neuroimage.2005.01.028

Stamatakis, E. A., Shafto, M. A., Williams, G., Tam, P., and Tyler, L. K. (2011). White matter changes and word finding failures with increasing age. PLoS One 6:e14496. doi: 10.1371/journal.pone.0014496

Sul, B., Lee, K. B., Hong, B. Y., Kim, J. S., Kim, J., Hwang, W. S., et al. (2019). Association of Lesion Location With Long-Term Recovery in Post-stroke Aphasia and Language Deficits. Front. Neurol. 10:776. doi: 10.3389/fneur.2019. 00776 
Sullivan, E. V., Adalsteinsson, E., Hedehus, M., Ju, C., Moseley, M., Lim, K. O., et al. (2001). Equivalent disruption of regional white matter microstructure in ageing healthy men and women. Neuroreport 12, 99-104. doi: 10.1097/ 00001756-200101220-00027

Sullivan, E. V., and Pfefferbaum, A. (2006). Diffusion tensor imaging and aging. Neurosci. Biobehav. Rev. 30, 749-761. doi: 10.1016/j.neubiorev.2006.06.002

Sullivan, E. V., Rohlfing, T., and Pfefferbaum, A. (2010). Quantitative fiber tracking of lateral and interhemispheric white matter systems in normal aging: relations to timed performance. Neurobiol. Aging 31, 464-481. doi: 10.1016/j. neurobiolaging.2008.04.007

Sundaram, S. K., Kumar, A., Makki, M. I., Behen, M. E., Chugani, H. T., and Chugani, D. C. (2008). Diffusion tensor imaging of frontal lobe in autism spectrum disorder. Cereb. Cortex 18, 2659-2665. doi: 10.1093/cercor/ bhn031

Teubner-Rhodes, S., Vaden, K. I., Cute, S. L., Yeatman, J. D., Dougherty, R. F., and Eckert, M. A. (2016). Aging-Resilient Associations between the Arcuate Fasciculus and Vocabulary Knowledge: microstructure or Morphology?. J. Neurosci. 36, 7210-7222. doi: 10.1523/JNEUROSCI.4342-15.2016

Thomason, M. E., and Thompson, P. M. (2011). Diffusion imaging, white matter, and psychopathology. Annu. Rev. Clin. Psychol. 7, 63-85. doi: 10.1146/annurevclinpsy-032210-104507

Tombaugh, T. N., Kozak, J., and Rees, L. (1999). Normative data stratified by age and education for two measures of verbal fluency: FAS and animal naming. Arch. Clin. Neuropsychol. 14, 167-177.

Troutman, S. B. W., and Diaz, M. T. (2020). White matter disconnection is related to age-related phonological deficits. Brain Imaging Behav. 14, 1555-1565. doi: 10.1007/s11682-019-00086-8

Vaqué-Alcázar, L., Sala-Llonch, R., Abellaneda-Pérez, K., Coll-Padrós, N., VallsPedret, C., Bargalló, N., et al. (2020). Functional and structural correlates of working memory performance and stability in healthy older adults. Brain Struct. Funct. 225, 375-386. doi: 10.1007/s00429-019-02009-1

Webb, C. E., Rodrigue, K. M., Hoagey, D. A., Foster, C. M., and Kennedy, K. M. (2020). Contributions of White Matter Connectivity and BOLD Modulation to Cognitive Aging: a Lifespan Structure-Function Association Study. Cereb. Cortex 30, 1649-1661. doi: 10.1093/cercor/bhz193

Westlye, L. T., Walhovd, K. B., Dale, A. M., Bjørnerud, A., Due-Tønnessen, P., Engvig, A., et al. (2010). Life-span changes of the human brain white matter: diffusion tensor imaging (DTI) and volumetry. Cereb. Cortex 20, 2055-2068. doi: $10.1093 /$ cercor/bhp280

Wingfield, A., and Grossman, M. (2006). Language and the aging brain: patterns of neural compensation revealed by functional brain imaging. J. Neurophysiol. 96, 2830-2839. doi: 10.1152/jn.00628.2006

Yogarajah, M., Focke, N. K., Bonelli, S. B., Thompson, P., Vollmar, C., McEvoy, A. W., et al. (2010). The structural plasticity of white matter networks following anterior temporal lobe resection. Brain 133, 2348-2364. doi: 10.1093/brain/ awq175

Zahr, N. M., Rohlfing, T., Pfefferbaum, A., and Sullivan, E. V. (2009). Problem solving, working memory, and motor correlates of association and commissural fiber bundles in normal aging: a quantitative fiber tracking study. Neuroimage 44, 1050-1062. doi: 10.1016/j.neuroimage.2008.09.046

Zhang, H., Sachdev, P. S., Wen, W., Kochan, N. A., Crawford, J. D., Brodaty, H., et al. (2013). Grey matter correlates of three language tests in non-demented older adults. PLoS One 8:e80215. doi: 10.1371/journal.pone.0080215

Zhang, Y., Du, A. T., Hayasaka, S., Jahng, G. H., Hlavin, J., Zhan, W., et al. (2010). Patterns of age-related water diffusion changes in human brain by concordance and discordance analysis. Neurobiol. Aging 31, 1991-2001. doi: 10.1016/j.neurobiolaging.2008.10.009

Conflict of Interest: The authors declare that the research was conducted in the absence of any commercial or financial relationships that could be construed as a potential conflict of interest.

Publisher's Note: All claims expressed in this article are solely those of the authors and do not necessarily represent those of their affiliated organizations, or those of the publisher, the editors and the reviewers. Any product that may be evaluated in this article, or claim that may be made by its manufacturer, is not guaranteed or endorsed by the publisher.

Copyright (C) 2021 Yeske, Hou, Adluru, Nair and Prabhakaran. This is an open-access article distributed under the terms of the Creative Commons Attribution License (CC BY). The use, distribution or reproduction in other forums is permitted, provided the original author(s) and the copyright owner(s) are credited and that the original publication in this journal is cited, in accordance with accepted academic practice. No use, distribution or reproduction is permitted which does not comply with these terms. 